\title{
Inviting Others to Life in Reciprocal Space
}

\author{
C Lind-Kovacs ${ }^{1}$ \\ ${ }^{1}$ Dept of Chemistry \& Biochemistry, Univ of Toledo, Toledo, $\mathrm{OH}$ \\ cora.lind@utoledo.edu
}

Crystallography is an important science that impacts many areas of research. The number of users of crystallographic techniques has increased significantly over the past decades, as diffractometers have become readily available, and many commercial programs with user-friendly interfaces have been developed. The growth in computing power has led to the development of advanced methods that were unthinkable in the past. Yet at the same time, formal crystallography teaching has declined, with many programs no longer offering formal crystallography classes. This combination has resulted in many avoidable errors in data collection, processing and interpretation of results. Making crystallographic teaching, both of theoretical concepts and hands-on training in data processing and analysis, available to the next generations of crystallographers is a crucial mandate. This talk gives a glimpse at several possible avenues to invite newcomers to a successful life in reciprocal space.

Acta Cryst. (2020). A76, a11 